\title{
Novel Concepts of Bipolar Fuzzy BCK-Submodules
}

\author{
M. A. Alghamdi, ${ }^{1,2}$ N. M. Muthana, ${ }^{1}$ and N. O. Alshehri ${ }^{1}$ \\ ${ }^{1}$ Department of Mathematics, Faculty of Sciences, King Abdulaziz University, Jeddah, Saudi Arabia \\ ${ }^{2}$ Department of Mathematics, Faculty of Sciences, University of Dammam, Dammam, Saudi Arabia
}

Correspondence should be addressed to M. A. Alghamdi; malghamdi0612@stu.kau.edu.sa

Received 3 September 2016; Accepted 7 November 2016; Published 23 January 2017

Academic Editor: Juan R. Torregrosa

Copyright (C) 2017 M. A. Alghamdi et al. This is an open access article distributed under the Creative Commons Attribution License, which permits unrestricted use, distribution, and reproduction in any medium, provided the original work is properly cited.

Translations and multiplications of bipolar fuzzy BCK-submodules are discussed. Extensions of bipolar fuzzy BCK-submodules are introduced. Relations between translations and multiplications of bipolar fuzzy BCK-submodules are presented.

\section{Introduction}

Fuzzy set theory was first proposed by Zadeh [1] as a means of handling uncertainty that is due to imprecision or vagueness rather than to randomness. It permits the gradual assessment of the membership of elements in a set, where every element is given a degree of membership between 0 and 1 .

As an extension of the traditional fuzzy sets, Lee [2] introduced the concept of bipolar-valued fuzzy sets where the membership degree range is enlarged from $[0,1]$ to $[-1,1]$. Bipolarity is important to distinguish between the following: (i) positive information that shows how much elements satisfy an associated property to the fuzzy set and (ii) negative information that demonstrates the satisfaction degree of elements to a counterproperty of the concerned fuzzy set. Many researchers were interested in studying bipolar-valued fuzzy sets and they applied them on several algebraic structures such as the following: BCK-algebras [3], hemirings [4], and semirings [5].

The notion of BCK-modules was presented by Abujabal et al. [6] in 1994. As a sequel of this work in field of the fuzzy set theory, Bakhshi [7] introduced the concept of fuzzy BCKsubmodules and investigated their properties. Later, generalized fuzzy BCK-submodules were formulated by other researchers [8] with some obtained basic properties.

Recently, M. A. Alghamdi, N. O. Alshehri, and N. M. Muthana introduced the notion of bipolar fuzzy BCKsubmodules and discussed many related results.
In this paper, we study translations, extensions, and multiplications of bipolar fuzzy BCK-submodules and investigate relations between them.

\section{Preliminaries}

In this section we provide basic definitions and results regarding BCK-algebras (modules), bipolar fuzzy BCK-submodules, and BCK-module homomorphisms.

By a BCK-algebra, we mean an algebra $(X, *, 0)$ of type $(2,0)$ satisfying the following axioms:

(I) $((x * y) *(x * z))(z * y)=0$

(II) $(x *(x * y)) * y=0$

(III) $x * x=0$

(IV) $0 * x=0$

(V) $x * y=0$ and $y * x=0$ implies $x=y$, for all $x, y, z \in X$

Let $(X, *, 0)$ be a BCK-algebra. Then $X$ is a partially ordered set with the partial ordering $\leq$ defined on $X$ by $x \leq y$ if and only if $x * y=0$. $X$ is said to be bounded if there is an element $1 \in X$ such that $x \leq 1$ for all $x \in X$. $X$ is said to be commutative (implicative) if $x \wedge y=y \wedge x(x *(y * x)=x)$ for all $x, y \in X$, where $x \wedge y=y *(y * x)$.

Definition 1 (see [6]). Let $X$ be a BCK-algebra. Then by a left $X$-module (abbreviated $X$-module) we mean an abelian 
group $M$ with an operation $X \times M \rightarrow M$ with $(x, m) \mapsto x m$ satisfies the following axioms for all $x, y \in X$ and $m, n \in M$ :
(1) $(x \wedge y) m=x(y m)$
(2) $x(m+n)=x m+x n$
(3) $0 m=0$

Moreover, if $X$ is bounded and $M$ satisfies $1 m=m$, for all $m \in M$, then $M$ is said to be unitary.

Example 2. Any bounded implicative BCK-algebra $X$ forms an $X$-module, where “ + " is defined as $x+y=(x * y) \vee(y * x)$ and $x y=x \wedge y$.

A subgroup $N$ of an $X$-module $M$ is called submodule of $M$ if $N$ is also an $X$-module.

Theorem 3 (see [7]). A subset $N$ of a BCK-module $M$ is a $B C K$-submodule of $M$ if and only if $n_{1}-n_{2} \in N$ and $x n \in N$ for all $n_{1}, n_{2}, n \in N$ and $x \in X$.

Definition 4 (see [6]). Let $M, N$ be modules over a BCKalgebra $X$. A mapping $f: M \rightarrow N$ is called $X$-homomorphism if

(1) $f\left(m_{1}+m_{2}\right)=f\left(m_{1}\right)+f\left(m_{2}\right)$;

(2) $f(x m)=x f(m)$ for all $m_{1}, m_{2}, m \in M$ and $x \in X$.

A BCK-module homomorphism is said to be monomorphism (epimorphism) if it is one to one (onto). If it is both one to one and onto, then we say that it is an isomorphism.

Let $X$ be the universe of discourse. A bipolar-valued fuzzy set (abbreviated bipolar fuzzy set) $\Phi$ of $X$ is an object having the form

$$
\Phi=\left\{\left(x ; \Phi^{+}(x), \Phi^{-}(x)\right) \mid x \in X\right\}
$$

where $\Phi^{+}: X \rightarrow[0,1]$ and $\Phi^{-}: X \rightarrow[-1,0]$ are mappings. The positive membership degree $\Phi^{+}(x)$ denotes the satisfaction degree of an element $x$ to the property corresponding to a bipolar-valued fuzzy set $\Phi=\left\{\left(x ; \Phi^{+}(x), \Phi^{-}(x)\right) \mid x \in\right.$ $X\}$, and the negative membership degree $\Phi^{-}(x)$ denotes the satisfaction degree of $x$ to some implicit counterproperty of $\Phi=\left\{\left(x ; \Phi^{+}(x), \Phi^{-}(x)\right) \mid x \in X\right\}$.

We shall use the symbol $\Phi=\left(X ; \Phi^{+}, \Phi^{-}\right)$for the bipolar fuzzy set $\Phi=\left\{\left(x ; \Phi^{+}(x), \Phi^{-}(x)\right) \mid x \in X\right\}$.

For a bipolar fuzzy set $\Phi=\left(X ; \Phi^{+}, \Phi^{-}\right)$and $(t, s) \in[0,1] \times$ $[-1,0]$, we define

$$
\begin{aligned}
& P(\Phi ; t)=\left\{x \in X \mid \Phi^{+}(x) \geq t\right\}, \\
& N(\Phi ; s)=\left\{x \in X \mid \Phi^{-}(x) \leq s\right\},
\end{aligned}
$$

which are called the positive $t$-level cut of $\Phi=\left(X ; \Phi^{+}, \Phi^{-}\right)$ and the negative $s$-level cut of $\Phi=\left(X, \Phi^{+}, \Phi^{-}\right)$, respectively. For $k \in[0,1]$, the set

$$
L(\Phi ; k)=P(\Phi ; k) \cap N(\Phi ;-k)
$$

is called the $k$-level cut of $\Phi=\left(X ; \Phi^{+}, \Phi^{-}\right)$(see [3]).
Definition 5 (see [9]). Let $\Phi=\left(X ; \Phi^{+}, \Phi^{-}\right)$and $\Psi=$ $\left(X ; \Psi^{+}, \Psi^{-}\right)$be bipolar fuzzy sets of $X$. If $\Psi^{+}(x) \geq \Phi^{+}(x)$ and $\Psi^{-}(x) \leq \Phi^{-}(x)$ for all $x \in X$, then we say that $\Psi=$ $\left(X ; \Psi^{+}, \Psi^{-}\right)$is a bipolar fuzzy extension of $\Phi=\left(X ; \Phi^{+}, \Phi^{-}\right)$ (simply $\Phi$ is subset of $\Psi$ ) and we write $\Phi \subseteq \Psi$.

Definition 6. A bipolar fuzzy set $\Phi=\left(M ; \Phi^{+}, \Phi^{-}\right)$of a BCKmodule $M$ is said to be a bipolar fuzzy BCK-submodule if it satisfies

$(\mathrm{BFS} 1) \Phi^{+}\left(m_{1}+m_{2}\right) \geq \min \left\{\Phi^{+}\left(m_{1}\right), \Phi^{+}\left(m_{2}\right)\right\}$ and

$$
\Phi^{-}\left(m_{1}+m_{2}\right) \leq \max \left\{\Phi^{-}\left(m_{1}\right), \Phi^{-}\left(m_{2}\right)\right\},
$$

(BFS2) $\Phi^{+}(-m)=\Phi^{+}(m)$ and $\Phi^{-}(-m)=\Phi^{-}(m)$,

$(\mathrm{BFS} 3) \Phi^{+}(x m) \geq \Phi^{+}(m)$ and $\Phi^{-}(x m) \leq \Phi^{-}(m)$.

For the sake of simplicity, we shall use the symbols $\mathscr{B} \mathscr{F}(M)$ and $\mathscr{B} \mathscr{F} \mathcal{S}(M)$ for the set of all bipolar fuzzy sets of $M$ and the set of all bipolar fuzzy BCK-submodules of $M$, respectively.

Theorem 7. A bipolar fuzzy set $\Phi=\left(M ; \Phi^{+}, \Phi^{-}\right) \in$ $\mathscr{B} \mathscr{F} \mathcal{S}(M)$ if and only if

(i) $\Phi^{+}(x m) \geq \Phi^{+}(m)$ and $\Phi^{-}(x m) \leq \Phi^{-}(m)$,

(ii) $\Phi^{+}\left(m_{1}-m_{2}\right) \geq \min \left\{\Phi^{+}\left(m_{1}\right), \Phi^{+}\left(m_{2}\right)\right\}$ and $\Phi^{-}\left(m_{1}-\right.$ $\left.m_{2}\right) \leq \max \left\{\Phi^{-}\left(m_{1}\right), \Phi^{-}\left(m_{2}\right)\right\}$ for all $m, m_{1}, m_{2} \in M$ and $x \in X$.

Theorem 8. Let $\Phi=\left(M ; \Phi^{+}, \Phi^{-}\right) \in \mathscr{B} \mathscr{F}(M)$. Then $\Phi \in$ $\mathscr{B} \mathscr{F} \mathcal{S}(M)$ if and only if

$$
\begin{aligned}
& \varnothing \neq P\left(\Phi^{+}, t\right) \quad \text { submodules of } M, \\
& \varnothing \neq N\left(\Phi^{-}, s\right) \quad \text { submodules of } M,
\end{aligned}
$$

for all $(t, s) \in[0,1] \times[-1,0]$.

Definition 9. Let $f: M \rightarrow N$ be a BCK-module homomorphism and let $\Phi \in \mathscr{B} \mathscr{F}(M)$ be a bipolar fuzzy set on $M$. Then the image of $f(\Phi)=\left(N ; f\left(\Phi^{+}\right), f\left(\Phi^{-}\right)\right)$of $\Phi$ under $f$ is defined as follows:

$$
\begin{aligned}
& f\left(\Phi^{+}\right)(n)= \begin{cases}\sup _{m \in f^{-1}(n)} \Phi^{+}(m), & \text { if } f^{-1}(n) \neq \varnothing \\
0, & \text { if } f^{-1}(n)=\varnothing,\end{cases} \\
& f\left(\Phi^{-}\right)(n)= \begin{cases}\inf _{m \in f^{-1}(n)} \Phi^{-}(m), & \text { if } f^{-1}(n) \neq \varnothing \\
0, & \text { if } f^{-1}(n)=\varnothing .\end{cases}
\end{aligned}
$$
$f$.

We shall call $f(\Phi)$ the homomorphic image of $\Phi$ under

Theorem 10. Let $f: M \rightarrow N$ be a BCK-module epimorphism and let $\Phi=\left(M ; \Phi^{+}, \Phi^{-}\right)$be a bipolar fuzzy BCK-submodule of $M$.Then the homomorphic image $f(\Phi)$ of $\Phi$ under $f$ is a bipolar fuzzy BCK-submodule on $\mathrm{N}$. 
Definition 11. Let $f: M \rightarrow N$ be a homomorphism of BCKmodules and $\Psi=\left(N ; \Psi^{+}, \Psi^{-}\right)$a bipolar fuzzy set of $N$. Then the inverse image of $\Psi, f^{-1}(\Psi)=\left(M ; f^{-1}\left(\Psi^{+}\right), f^{-1}\left(\Psi^{-}\right)\right)$, is the bipolar fuzzy set on $M$ given by $f^{-1}\left(\Psi^{+}\right)(m)=\Psi^{+}(f(m))$ and $f^{-1}\left(\Psi^{-}\right)(m)=\Psi^{-}(f(m))$ for all $m \in M$.

Theorem 12. Let $f: M \rightarrow N$ be a homomorphism of BCK-modules and $\Psi=\left(N ; \Psi^{+}, \Psi^{-}\right) \in \mathscr{B} \mathscr{F} \mathcal{S}(N)$, and then the inverse image $f^{-1}(\Psi)=\left(M ; f^{-1}\left(\Psi^{+}\right), f^{-1}\left(\Psi^{-}\right)\right) \in$ $\mathscr{B} \mathscr{F} \mathcal{S}(M)$.

Theorem 13. Let $f: M \rightarrow N$ be an epimorphism of BCKmodules. If $\Psi=\left(N ; \Psi^{+}, \Psi^{-}\right)$is a bipolar fuzzy set on $N$ such that the inverse image $f^{-1}(\Psi)=\left(M ; f^{-1}\left(\Psi^{+}\right), f^{-1}\left(\Psi^{-}\right)\right) \in$ $\mathscr{B} \mathscr{F} \mathcal{S}(M)$, then $\Psi \in \mathscr{B} \mathscr{F} \mathcal{S}(N)$.

\section{Fuzzy Translations and Fuzzy Multiplications of $\mathscr{B} \mathscr{F} \mathcal{S}(M)$}

Newly, many researchers have studied fuzzy translations and fuzzy multiplications and their effects on several types of fuzzy sets. Lee and others [10] discussed fuzzy translations and multiplications in the case of fuzzy subalgebras. Bipolar fuzzy translations in BCK/BCI algebras were introduced by Jun et al. [9]. Later, Jun [11] investigated translations of fuzzy ideals of BCK-algebras. In this section, we present fuzzy translations and multiplications of bipolar fuzzy BCKsubmodules and discuss related properties.

In what follows, $M$ and $N$ are considered to be modules over some BCK-algebra $X$, unless otherwise specified.

For any bipolar fuzzy set $\Phi=\left(M ; \Phi^{+}, \Phi^{-}\right)$on $M$, we denote

$$
\begin{aligned}
& \top:=1-\sup \left\{\Phi^{+}(m) \mid m \in M\right\}, \\
& \perp:=-1-\inf \left\{\Phi^{-}(m) \mid m \in M\right\} .
\end{aligned}
$$

Definition 14. Let $\Phi=\left(M ; \Phi^{+}, \Phi^{-}\right)$be a bipolar fuzzy set on $M$ and $(\alpha, \beta) \in[0, \top] \times[\perp, 0]$. By a bipolar fuzzy $(\alpha, \beta)$ translation of $\Phi$ we mean a bipolar fuzzy set $T_{(\alpha, \beta)}(\Phi)=(M$; $\left.T_{\alpha}\left(\Phi^{+}\right), T_{\beta}\left(\Phi^{-}\right)\right)$, where

$$
\begin{aligned}
& T_{\alpha}\left(\Phi^{+}\right): M \longrightarrow[0,1], \quad m \longmapsto \Phi^{+}(m)+\alpha, \\
& T_{\beta}\left(\Phi^{-}\right): M \longrightarrow[-1,0], \quad m \longmapsto \Phi^{-}(m)+\beta .
\end{aligned}
$$

Theorem 15. Let $\Phi=\left(M ; \Phi^{+}, \Phi^{-}\right) \in \mathscr{B} \mathscr{F} \mathcal{S}(M)$ and $(\alpha, \beta) \in[0, \top] \times[\perp, 0]$. Then the $(\alpha, \beta)$-translation $T_{(\alpha, \beta)}(\Phi)=$ $\left(M ; T_{\alpha}\left(\Phi^{+}\right), T_{\beta}\left(\Phi^{-}\right)\right) \in \mathscr{B} \mathscr{F} \mathcal{S}(M)$.

Proof. Let $m, m_{1}, m_{2} \in M$ and $x \in X$. Then

$$
\begin{aligned}
T_{\alpha} & \left(\Phi^{+}\right)\left(m_{1}-m_{2}\right)=\Phi^{+}\left(m_{1}-m_{2}\right)+\alpha \\
& \geq \min \left\{\Phi^{+}\left(m_{1}\right), \Phi^{+}\left(m_{2}\right)\right\}+\alpha \\
& =\min \left\{\Phi^{+}\left(m_{1}\right)+\alpha, \Phi^{+}\left(m_{2}\right)+\alpha\right\}
\end{aligned}
$$

$$
\begin{aligned}
& =\min \left\{T_{\alpha}\left(\Phi^{+}\right)\left(m_{1}\right), T_{\alpha}\left(\Phi^{+}\right)\left(m_{2}\right)\right\}, \\
T_{\alpha} & \left(\Phi^{+}\right)(x m)=\Phi^{+}(x m)+\alpha \geq \Phi^{+}(m)+\alpha \\
& =T_{\alpha}\left(\Phi^{+}\right)(m) .
\end{aligned}
$$

Analogously,

$$
\begin{aligned}
& T_{\beta}\left(\Phi^{-}\right)\left(m_{1}-m_{2}\right) \\
& \quad \leq \max \left\{T_{\beta}\left(\Phi^{-}\right)\left(m_{1}\right), T_{\beta}\left(\Phi^{-}\right)\left(m_{2}\right)\right\}, \\
& T_{\beta}\left(\Phi^{-}\right)(x m) \leq T_{\beta}\left(\Phi^{-}\right)(m) .
\end{aligned}
$$

Hence $T_{(\alpha, \beta)}(\Phi)$ is a bipolar fuzzy BCK-submodule on $M$.

Theorem 16. Let $\Phi=\left(M ; \Phi^{+}, \Phi^{-}\right) \in \mathscr{B} \mathscr{F}(M)$ such that the bipolar fuzzy $(\alpha, \beta)$-translation $T_{(\alpha, \beta)}(\Phi)=\left(M ; T_{\alpha}\left(\Phi^{+}\right)\right.$, $\left.T_{\beta}\left(\Phi^{-}\right)\right)$of $\Phi=\left(M ; \Phi^{+}, \Phi^{-}\right)$is a bipolar fuzzy BCK-submodule on $M$ for some $(\alpha, \beta) \in[0, \top] \times[\perp, 0]$. Then $\Phi=(M$; $\left.\Phi^{+}, \Phi^{-}\right) \in \mathscr{B} \mathscr{F} \mathcal{S}(M)$.

Proof. Assume that $T_{(\alpha, \beta)}(\Phi)=\left(M ; T_{\alpha}\left(\Phi^{+}\right), T_{\beta}\left(\Phi^{-}\right)\right) \in$ $\mathscr{B} \mathscr{F} \mathcal{S}(M)$ for some $(\alpha, \beta) \in[0, \top] \times[\perp, 0]$. For any $m, m_{1}$, $m_{2} \in M$ and $x \in X$, we have

$$
\begin{aligned}
\Phi^{+}(x m)+\alpha & =T_{\alpha}\left(\Phi^{+}\right)(x m) \geq T_{\alpha}\left(\Phi^{+}\right)(m) \\
& =\Phi^{+}(m)+\alpha
\end{aligned}
$$

that is,

$$
\Phi^{+}(x m) \geq \Phi^{+}(m) .
$$

Moreover,

$$
\begin{gathered}
\Phi^{+}\left(m_{1}-m_{2}\right)+\alpha=T_{\alpha}\left(\Phi^{+}\right)\left(m_{1}-m_{2}\right) \\
\geq \min \left\{T_{\alpha}\left(\Phi^{+}\right)\left(m_{1}\right), T_{\alpha}\left(\Phi^{+}\right)\left(m_{2}\right)\right\} \\
=\min \left\{\Phi^{+}\left(m_{1}\right)+\alpha, \Phi^{+}\left(m_{2}\right)+\alpha\right\} \\
=\min \left\{\Phi^{+}\left(m_{1}\right), \Phi^{+}\left(m_{2}\right)\right\}+\alpha,
\end{gathered}
$$

which implies that $\Phi^{+}\left(m_{1}-m_{2}\right) \geq \min \left\{\Phi^{+}\left(m_{1}\right), \Phi^{+}\left(m_{2}\right)\right\}$. By similar argument, we have $\Phi^{-}\left(m_{1}-m_{2}\right) \leq \max \left\{\Phi^{-}\left(m_{1}\right)\right.$, $\left.\Phi^{-}\left(m_{2}\right)\right\}$ and $\Phi^{-}(x m) \leq \Phi^{-}(m)$. Hence $\Phi=\left(M ; \Phi^{+}, \Phi^{-}\right) \epsilon$ $\mathscr{B} \mathscr{F} \mathcal{S}(M)$.

Definition 17. Let $\Phi=\left(M ; \Phi^{+}, \Phi^{-}\right)$and $\Psi=\left(M ; \Psi^{+}, \Psi^{-}\right)$be bipolar fuzzy sets on $M$. Then $\Psi=\left(M ; \Psi^{+}, \Psi^{-}\right)$is called a bipolar fuzzy s-extension of $\Phi=\left(M ; \Phi^{+}, \Phi^{-}\right)$if the following assertions hold:

(i) $\Psi=\left(M ; \Psi^{+}, \Psi^{-}\right)$is a bipolar fuzzy extension of $\Phi=$ $\left(M ; \Phi^{+}, \Phi^{-}\right)$

(ii) If $\Phi=\left(M ; \Phi^{+}, \Phi^{-}\right)$is a bipolar fuzzy BCK-submodule of $M$, then so is $\Psi=\left(M ; \Psi^{+}, \Psi^{-}\right)$. 
By means of the definition of $(\alpha, \beta)$-translation, we know that $T_{\alpha}\left(\Phi^{+}\right)(m) \geq \Phi^{+}(m)$ and $T_{\beta}\left(\Phi^{-}\right)(m) \leq \Phi^{-}(m)$ for all $m \in M$. Hence, according to Theorem 15, we have the following theorem.

Theorem 18. Let $\Phi=\left(M ; \Phi^{+}, \Phi^{-}\right)$be a bipolar fuzzy BCKsubmodule of $M$ and $(\alpha, \beta) \in[0, \top] \times[\perp, 0]$. Then the bipolar fuzzy $(\alpha, \beta)$-translation $T_{(\alpha, \beta)}(\Phi)=\left(M ; T_{\alpha}\left(\Phi^{+}\right), T_{\beta}\left(\Phi^{-}\right)\right)$is a bipolar fuzzy s-extension of $\Phi=\left(M ; \Phi^{+}, \Phi^{-}\right)$.

The converse of Theorem 18 is not true in general as seen in the following example.

Example 19. Let $X=\{0, a, b, c, d\}$ along with a binary operation $*$ defined in $(14)$, and then $(X, *, 0)$ forms an unbounded commutative BCK-algebra. Let $M=\{0, a, b, c\}$ be a subset of $X$ with binary operation + defined by $x+y=(x * y) \vee(y * x)$. Then $M$ is a commutative group as shown in (15) (see [12]). Define scalar multiplication $(X, M) \rightarrow X$ by $x m=x \wedge m$ for all $x \in X$ and $m \in M$ that is given in (16). Consequently, $M$ forms an $X$-module.

\begin{tabular}{|c|c|c|c|c|c|}
\hline$*$ & 0 & $a$ & $b$ & $c$ & $d$ \\
\hline 0 & 0 & 0 & 0 & 0 & 0 \\
\hline$a$ & $a$ & 0 & $a$ & 0 & $a$ \\
\hline$b$ & $b$ & $b$ & 0 & 0 & $b$ \\
\hline$c$ & $c$ & $b$ & $a$ & 0 & $b$ \\
\hline$d$ & $d$ & $a$ & $d$ & $a$ & 0 \\
\hline
\end{tabular}

$$
\begin{array}{|c|c|c|c|c|}
\hline+ & 0 & a & b & c \\
\hline 0 & 0 & a & b & c \\
\hline a & a & 0 & c & b \\
\hline b & b & c & 0 & a \\
\hline c & c & b & a & 0 \\
\hline
\end{array}
$$

$$
\begin{array}{|l|l|l|l|l|}
\hline \wedge & 0 & a & b & c \\
\hline 0 & 0 & 0 & 0 & 0 \\
\hline a & 0 & a & 0 & a \\
\hline b & 0 & b & b & b \\
\hline c & 0 & a & b & c \\
\hline d & 0 & c & 0 & c \\
\hline
\end{array}
$$

Define a bipolar fuzzy set $\Phi=\left(M ; \Phi^{+}, \Phi^{-}\right)$on $M$ as shown in

\begin{tabular}{|c|c|c|c|c|}
\hline$M$ & 0 & $a$ & $b$ & $c$ \\
\hline$\Phi^{+}$ & 0.8 & 0.6 & 0.6 & 0.6 \\
\hline$\Phi^{-}$ & -0.9 & -0.5 & -0.5 & -0.5 \\
\hline
\end{tabular}

Then $\Phi$ is a bipolar fuzzy BCK-submodule on $M$. Let $\Psi=$ $\left(M ; \Psi^{+}, \Psi^{-}\right)$be a bipolar fuzzy set on $M$ given by

\begin{tabular}{c|c|c|c|c|}
\hline$M$ & 0 & $a$ & $b$ & $c$ \\
\hline$\Psi^{+}$ & 0.9 & 0.8 & 0.8 & 0.8 \\
\hline$\Psi^{-}$ & -0.9 & -0.6 & -0.6 & -0.6 \\
\hline
\end{tabular}

Then $\Psi$ is also a bipolar fuzzy BCK-submodule on $M$. Since $\Psi^{+}(m) \geq \Phi^{+}(m)$ and $\Psi^{-}(m) \leq \Phi^{-}(m)$ for all $m \in M$, hence $\Psi$ is a bipolar fuzzy $s$-extension of $\Phi$. But it is not a bipolar fuzzy $(\alpha, \beta)$-translation of $\Phi$ for all $(\alpha, \beta) \in[0, \top] \times[\perp$ $, 0]$.

For a bipolar fuzzy set $\Phi=\left(M ; \Phi^{+}, \Phi^{-}\right)$on $M$, consider the following two sets:

$$
\begin{aligned}
P_{\alpha}\left(\Phi^{+}, t\right) & :=\left\{m \in M \mid \Phi^{+}(m) \geq t-\alpha\right\} \\
N_{\beta}\left(\Phi^{-}, s\right) & :=\left\{m \in M \mid \Phi^{-}(m) \leq s-\beta\right\}
\end{aligned}
$$

where $(\alpha, \beta) \in[0, \top] \times[\perp, 0]$ and $(t, s) \in[\alpha, 1] \times[-1, \beta]$.

If $\Phi=\left(M ; \Phi^{+}, \Phi^{-}\right)$is a bipolar fuzzy BCK-submodule on $M$, then $P_{\alpha}\left(\Phi^{+} ; t\right)$ and $N_{\beta}\left(\Phi^{-} ; s\right)$ are submodules of $M$ for all $(t, s) \in \operatorname{Im}\left(\Phi^{+}\right) \times \operatorname{Im}\left(\Phi^{-}\right)$with $t \geq \alpha$ and $s \leq \beta$. However, if we do not give the condition that $\Phi=\left(M ; \Phi^{+}, \Phi^{-}\right)$is a bipolar fuzzy BCK-submodule on $M$, then at least one of $P_{\alpha}\left(\Phi^{+} ; t\right)$ and $N_{\beta}\left(\Phi^{-} ; s\right)$ may not be a submodule of $M$ as seen in the following example.

Example 20. Let $X=\{0, a, b, c, d\}$ be a commutative BCKalgebra with the binary operation $*$ defined by $(20)$. Let $M=\{0, a, b, c\}$ be a subset of $X$ along with an operation + defined by (21). Then $(M,+)$ is a commutative group. Define the action of $X$ on $M$ by $x m=x \wedge m$ that is shown in (22). Consequently, a routine exercise of calculations shows that $M$ forms an $X$-module (see [12]).

\begin{tabular}{|l|l|l|l|l|l|}
\hline$*$ & 0 & $a$ & $b$ & $c$ & $d$ \\
\hline 0 & 0 & 0 & 0 & 0 & 0 \\
\hline$a$ & $a$ & 0 & $a$ & 0 & $a$ \\
\hline$b$ & $b$ & $b$ & 0 & 0 & $b$ \\
\hline$c$ & $c$ & $b$ & $a$ & 0 & $d$ \\
\hline$d$ & $d$ & $d$ & $d$ & $d$ & 0 \\
\hline
\end{tabular}

\begin{tabular}{|l|l|l|l|l|}
\hline+ & 0 & $a$ & $b$ & $c$ \\
\hline 0 & 0 & $a$ & $b$ & $c$ \\
\hline$a$ & $a$ & 0 & $c$ & $b$ \\
\hline$b$ & $b$ & $c$ & 0 & $a$ \\
\hline$c$ & $c$ & $b$ & $a$ & 0 \\
\hline
\end{tabular}

\begin{tabular}{|l|l|l|l|l|}
\hline$\wedge$ & 0 & $a$ & $b$ & $c$ \\
\hline 0 & 0 & 0 & 0 & 0 \\
\hline$a$ & 0 & $a$ & 0 & $a$ \\
\hline$b$ & 0 & $b$ & $b$ & $b$ \\
\hline$c$ & 0 & $a$ & $b$ & $c$ \\
\hline$d$ & 0 & 0 & 0 & 0 \\
\hline
\end{tabular}

Equation (23) define a bipolar fuzzy set $\Phi=\left(M ; \Phi^{+}, \Phi^{-}\right)$ on $M$.

\begin{tabular}{|c|c|c|c|c|}
\hline$M$ & 0 & $a$ & $b$ & $c$ \\
\hline$\Phi^{+}$ & 0.9 & 0.6 & 0.3 & 0.4 \\
\hline$\Phi^{-}$ & -0.8 & -0.7 & -0.5 & -0.6 \\
\hline
\end{tabular}

For $\alpha=0.2$ and $t=0.6, P_{\alpha}\left(\Phi^{+} ; t\right)=\{0, a, c\}$ is not a submodule of $M$ since $a-c=b \notin P_{\alpha}\left(\Phi^{+} ; t\right)$. Note that $\Phi$ is not a bipolar fuzzy BCK-submodule on $M$ since $\Phi^{+}(a-c)=$ $\Phi^{+}(b)=0.3 \nsupseteq 0.4=\min \left\{\Phi^{+}(a), \Phi^{+}(c)\right\}$. 
Theorem 21. Let $\Phi=\left(M ; \Phi^{+}, \Phi^{-}\right)$be a bipolar fuzzy set on $M$ and $(\alpha, \beta) \in[0, \top] \times[\perp, 0]$. Then the $(\alpha, \beta)$-translation $T_{(\alpha, \beta)}(\Phi)=\left(M ; T_{\alpha}\left(\Phi^{+}\right), T_{\beta}\left(\Phi^{-}\right)\right)$of $\Phi=\left(M ; \Phi^{+}, \Phi^{-}\right)$is a bipolar fuzzy BCK-submodule of $M$ if and only if $P_{\alpha}\left(\Phi^{+} ; t\right)$ and $N_{\beta}\left(\Phi^{-} ; s\right)$ are submodules of $M$ for all $(t, s) \in \operatorname{Im}\left(\Phi^{+}\right) \times$ $\operatorname{Im}\left(\Phi^{-}\right)$with $t \geq \alpha$ and $s \leq \beta$.

Proof. Proof is immediately by Theorems 8, 15, and 16 .

Theorem 22. Let $\Phi=\left(M ; \Phi^{+}, \Phi^{-}\right)$be a bipolar fuzzy BCKsubmodule of $M$ and let $\alpha_{1}, \alpha_{2} \in[0, T]$ and $\beta_{1}, \beta_{2} \in[\perp$ ,0]. If $\alpha_{1} \geq \alpha_{2}$ and $\beta_{1} \leq \beta_{2}$, then the $\left(\alpha_{1}, \beta_{1}\right)$-translation $T_{\left(\alpha_{1}, \beta_{1}\right)}(\Phi)=\left(M ; T_{\alpha_{1}}\left(\Phi^{+}\right), T_{\beta_{1}}\left(\Phi^{-}\right)\right)$of $\Phi=\left(M ; \Phi^{+}\right.$, $\left.\Phi^{-}\right)$is a bipolar fuzzy s-extension of the $\left(\alpha_{2}, \beta_{2}\right)$-translation $T_{\left(\alpha_{2}, \beta_{2}\right)}(\Phi)=\left(M ; T_{\alpha_{2}}\left(\Phi^{+}\right), T_{\beta_{2}}\left(\Phi^{-}\right)\right)$.

Proof. It is straightforward.

Theorem 23. Let $\Phi=\left(M ; \Phi^{+}, \Phi^{-}\right)$be a bipolar fuzzy BCKsubmodule of $M$ and $(\alpha, \beta) \in[0, \top] \times[\perp, 0]$. For every bipolar fuzzy s-extension $\Psi=\left(M ; \Psi^{+}, \Psi^{-}\right)$of the $(\alpha, \beta)$-translation $T_{(\alpha, \beta)}(\Phi)=\left(M ; T_{\alpha}\left(\Phi^{+}\right), T_{\beta}\left(\Phi^{-}\right)\right)$, there exists $(k, h) \in[0, \top] \times$ $[\perp, 0]$ such that $k \geq \alpha$ and $h \leq \beta$, and $\Psi=\left(M ; \Psi^{+}, \Psi^{-}\right)$is a bipolar fuzzy s-extension of the $(k, h)$-translation $T_{(k, h)}(\Phi)=$ $\left(M ; T_{k}\left(\Phi^{+}\right), T_{h}\left(\Phi^{-}\right)\right)$.

Proof. Assume that $\Psi=\left(M ; \Psi^{+}, \Psi^{-}\right)$is a bipolar fuzzy $s^{-}$ extension of the $(\alpha, \beta)$-translation $T_{(\alpha, \beta)}(\Phi)=\left(M ; T_{\alpha}\left(\Phi^{+}\right)\right.$, $\left.T_{\beta}\left(\Phi^{-}\right)\right)$. Then $\Psi^{+}(m) \geq T_{\alpha}\left(\Phi^{+}\right)(m)=\Phi^{+}(m)+\alpha$ and $\Psi^{-}(m) \leq T_{\beta}\left(\Phi^{-}\right)(m)=\Phi^{-}(m)+\beta$. Pick $(k, h) \in[0, \top] \times[\perp, 0]$ such that $\Psi^{+}(m) \geq \Phi^{+}(m)+k \geq \Phi^{+}(m)+\alpha$ and $\Psi^{-}(m) \leq$ $\Phi^{-}(m)+h \leq \Phi^{-}(m)+\beta$. Then $\Psi=\left(M ; \Psi^{+}, \Psi^{-}\right)$is an extension of $T_{(k, h)}(\Phi)=\left(M ; T_{k}\left(\Phi^{+}\right), T_{h}\left(\Phi^{-}\right)\right)$and since $\Phi=$ $\left(M ; \Phi^{+}, \Phi^{-}\right)$is a bipolar fuzzy BCK-submodule of $M$, both of $\Psi=\left(M ; \Psi^{+}, \Psi^{-}\right)$and $T_{(k, h)}(\Phi)=\left(M ; T_{k}\left(\Phi^{+}\right), T_{h}\left(\Phi^{-}\right)\right)$ are bipolar fuzzy BCK-submodules of $M$. Therefore $\Psi=$ $\left(M ; \Psi^{+}, \Psi^{-}\right)$is a bipolar fuzzy $s$-extension of the $(k, h)$ translation $T_{(k, h)}(\Phi)=\left(M ; T_{k}\left(\Phi^{+}\right), T_{h}\left(\Phi^{-}\right)\right)$.

Theorem 23 is illustrated by the following example.

Example 24. Let $X=\{0, a, b, c, d, 1\}$ be a set along with binary operation $*$ defined by (24). Then $X$ is a bounded commutative BCK-algebra. Let $M=\{0, a, c, d\}$ and define + on $M$ as $x+y=(x * y) \vee(y * x)$ which is given in (25). Then $(M,+)$ is a commutative group. Quick calculations reveal that $M$ is an $X$-module under the operation $x m=x \wedge m$ shown in (26) (see [12]):

$$
\begin{array}{|c|c|c|c|c|c|c|}
\hline * & 0 & a & b & c & d & 1 \\
\hline 0 & 0 & 0 & 0 & 0 & 0 & 0 \\
\hline a & a & 0 & 0 & a & 0 & 0 \\
\hline b & b & a & 0 & b & a & 0 \\
\hline c & c & c & c & 0 & 0 & 0 \\
\hline d & d & c & c & a & 0 & 0 \\
\hline 1 & 1 & d & c & b & a & 0 \\
\hline
\end{array}
$$

$$
\begin{array}{|l|l|l|l|l|}
\hline+ & 0 & a & c & d \\
\hline 0 & 0 & a & c & d \\
\hline a & a & 0 & d & c \\
\hline c & c & d & 0 & a \\
\hline d & d & c & a & 0 \\
\hline
\end{array}
$$

\begin{tabular}{|l|l|l|l|l|}
$\wedge$ & 0 & $a$ & $c$ & $d$ \\
\hline 0 & 0 & 0 & 0 & 0 \\
\hline$a$ & 0 & $a$ & 0 & $a$ \\
\hline$b$ & 0 & $a$ & 0 & $a$ \\
\hline$c$ & 0 & 0 & $c$ & $c$ \\
\hline$d$ & 0 & $a$ & $c$ & $d$ \\
\hline 1 & 0 & $a$ & $c$ & $d$ \\
\hline
\end{tabular}

Define a bipolar fuzzy set $\Phi=\left(M ; \Phi^{+}, \Phi^{-}\right)$on $M$ by

\begin{tabular}{|c|c|c|c|c|}
\hline$M$ & 0 & $a$ & $c$ & $d$ \\
\hline$\Phi^{+}$ & 0.7 & 0.3 & 0.3 & 0.3 \\
\hline$\Phi^{-}$ & -0.6 & -0.4 & -0.4 & -0.4 \\
\hline
\end{tabular}

Then $\Phi$ is a bipolar fuzzy BCK-submodule on $M$, and $\mathrm{T}=$ $0.3, \perp=-0.4$. If we take $\alpha=0.2$ and $\beta=-0.3$, then (28) shows the bipolar fuzzy $(\alpha, \beta)$-translation $T_{(\alpha, \beta)}(\Phi)$ of $\Phi$

\begin{tabular}{|c|c|c|c|c|}
\hline$M$ & 0 & $a$ & $c$ & $d$ \\
\hline$T_{\alpha}\left(\Phi^{+}\right)$ & 0.9 & 0.5 & 0.5 & 0.5 \\
\hline$T_{\beta}\left(\Phi^{-}\right)$ & -0.9 & -0.7 & -0.7 & -0.7 \\
\hline
\end{tabular}

Let $\Psi=\left(M ; \Psi^{+}, \Psi^{-}\right)$be a bipolar fuzzy set on $M$ defined by

\begin{tabular}{|c|c|c|c|c|}
\hline$M$ & 0 & $a$ & $c$ & $d$ \\
\hline$\Psi^{+}$ & 1 & 0.9 & 0.9 & 0.9 \\
\hline$\Psi^{-}$ & -1 & -0.9 & -0.9 & -0.9 \\
\hline
\end{tabular}

Clearly $\Psi$ is a bipolar fuzzy BCK-submodule on $M$ which is a bipolar fuzzy extension of $T_{(\alpha, \beta)}(\Phi)$. But $\Psi$ is not a bipolar fuzzy $(\alpha, \beta)$-translation of $\Phi$ for all $(\alpha, \beta) \in[0, \top] \times[\perp, 0]$. Take $k=0.3, h=-0.35$, and then $k>\alpha, h<\beta$, and the fuzzy $(k, h)$-translation $T_{(k, h)}(\Phi)$ of $\Phi$ is given by

\begin{tabular}{|c|c|c|c|c|}
\hline$M$ & 0 & $a$ & $c$ & $d$ \\
\hline$T_{k}\left(\Phi^{+}\right)$ & 1 & 0.6 & 0.6 & 0.6 \\
\hline$T_{h}\left(\Phi^{-}\right)$ & -0.95 & -0.75 & -0.75 & -0.75 \\
\hline
\end{tabular}

Note that $\Psi^{+}(m) \geq T_{k}\left(\Phi^{+}\right)$and $\Psi^{-}(m) \leq T_{h}\left(\Phi^{-}\right)(m)$ for all $m \in M$, and so $\Psi$ is a bipolar fuzzy $s$-extension of the bipolar fuzzy $(k, h)$-translation $T_{(k, h)}(\Phi)$ of $\Phi$.

Definition 25. Let $\Phi=\left(M ; \Phi^{+}, \Phi^{-}\right)$be a bipolar fuzzy set on $M$ and $\gamma, \delta \in[0,1]$. By a bipolar fuzzy $(\gamma, \delta)$-multiplication of $\Phi=\left(M ; \Phi^{+}, \Phi^{-}\right)$we mean a bipolar fuzzy set $\mathrm{m}_{(\gamma, \delta)}(\Phi)=$ $\left(M ; \mathrm{m}_{\gamma}\left(\Phi^{+}\right), \mathrm{m}_{\delta}\left(\Phi^{-}\right)\right)$, where

$$
\begin{aligned}
& \mathrm{m}_{\gamma}\left(\Phi^{+}\right)(m)=\Phi^{+}(m) \cdot \gamma, \\
& \mathrm{m}_{\delta}\left(\Phi^{-}\right)(m)=\Phi^{-}(m) \cdot \delta,
\end{aligned}
$$

for all $m \in M$. 
Theorem 26. If $\Phi=\left(M ; \Phi^{+}, \Phi^{-}\right) \in \mathscr{B} \mathscr{F} \mathcal{S}(M)$, then the bipolar fuzzy $(\gamma, \delta)$-multiplication $\mathrm{m}_{(\gamma, \delta)}(\Phi)=\left(M ; \mathrm{m}_{\gamma}\left(\Phi^{+}\right)\right.$, $\left.\mathrm{m}_{\delta}\left(\Phi^{-}\right)\right) \in \mathscr{B} \mathscr{F} \mathcal{S}(M)$ for all $\gamma, \delta \in[0,1]$.

Proof. Assume that $\Phi=\left(M ; \Phi^{+}, \Phi^{-}\right)$is a bipolar fuzzy BCKsubmodule on $M$, and let $m, m_{1}, m_{2} \in M$ and $x \in X$. Then

$$
\begin{aligned}
\mathrm{m}_{\gamma}\left(\Phi^{+}\right)(x m) & =\Phi^{+}(x m) \cdot \gamma \geq \Phi^{+}(m) \cdot \gamma \\
& =\mathrm{m}_{\gamma}\left(\Phi^{+}\right)(m), \\
\mathrm{m}_{\delta}\left(\Phi^{-}\right)(x m) & =\Phi^{-}(x m) \cdot \delta \leq \Phi^{-}(m) \cdot \delta \\
& =\mathrm{m}_{\delta}\left(\Phi^{-}\right)(m) .
\end{aligned}
$$

Moreover,

$$
\begin{aligned}
\mathrm{m}_{\gamma} & \left(\Phi^{+}\right)\left(m_{1}-m_{2}\right)=\Phi^{+}\left(m_{1}-m_{2}\right) \cdot \gamma \\
& \geq \min \left\{\Phi^{+}\left(m_{1}\right), \Phi^{+}\left(m_{2}\right)\right\} \cdot \gamma \\
& =\min \left\{\Phi^{+}\left(m_{1}\right) \cdot \gamma, \Phi^{+}\left(m_{2}\right) \cdot \gamma\right\} \\
& =\min \left\{\mathrm{m}_{\gamma}\left(\Phi^{+}\right)\left(m_{1}\right), \mathrm{m}_{\gamma}\left(\Phi^{+}\right)\left(m_{2}\right)\right\} \\
\mathrm{m}_{\delta} & \left(\Phi^{-}\right)\left(m_{1}-m_{2}\right)=\Phi^{-}\left(m_{1}-m_{2}\right) \cdot \delta \\
& \leq \max \left\{\Phi^{-}\left(m_{1}\right), \Phi^{-}\left(m_{2}\right)\right\} \cdot \delta \\
& =\max \left\{\Phi^{-}\left(m_{1}\right) \cdot \delta, \Phi^{-}\left(m_{2}\right) \cdot \delta\right\} \\
& =\max \left\{\mathrm{m}_{\delta}\left(\Phi^{-}\right)\left(m_{1}\right), \mathrm{m}_{\delta}\left(\Phi^{-}\right)\left(m_{2}\right)\right\} .
\end{aligned}
$$

Therefore $\mathrm{m}_{(\gamma, \delta)}(\Phi)=\left(M ; \mathrm{m}_{\gamma}\left(\Phi^{+}\right), \mathrm{m}_{\delta}\left(\Phi^{-}\right)\right)$is a bipolar fuzzy BCK-submodule on $M$.

Theorem 27. For any bipolar fuzzy set $\Phi=\left(M ; \Phi^{+}, \Phi^{-}\right)$on $M$, the following are equivalent:

(i) $\Phi=\left(M ; \Phi^{+}, \Phi^{-}\right)$is a bipolar fuzzy BCK-submodule on $M$.

(ii) $(\forall \gamma, \delta \in(0,1])\left(\mathrm{m}_{(\gamma, \delta)}(\Phi)=\left(M ; \mathrm{m}_{\gamma}\left(\Phi^{+}\right), \mathrm{m}_{\delta}\left(\Phi^{-}\right)\right)\right.$is a bipolar fuzzy BCK-submodule on $M)$.

Proof. By Theorem 27, (i) implies (ii). Now let $\gamma, \delta \in(0,1]$ be such that $\mathrm{m}_{(\gamma, \delta)}(\Phi)=\left(M ; \mathrm{m}_{\gamma}\left(\Phi^{+}\right), \mathrm{m}_{\delta}\left(\Phi^{-}\right)\right)$is a bipolar fuzzy BCK-submodule on $M$ and let $x \in X, m, m_{1}, m_{2} \in M$. Then

$$
\begin{aligned}
\Phi^{+}(x m) \cdot \gamma & =\mathrm{m}_{\gamma}\left(\Phi^{+}\right)(x m) \geq \mathrm{m}_{\gamma}\left(\Phi^{+}\right)(m) \\
& =\Phi^{+}(m) \cdot \gamma, \\
\Phi^{-}(m) \cdot \delta & =\mathrm{m}_{\delta}\left(\Phi^{-}\right)(x m) \leq \mathrm{m}_{\delta}\left(\Phi^{-}\right)(m) \\
& =\Phi^{-}(m) \cdot \delta,
\end{aligned}
$$

and since $\gamma \neq 0$ and $\delta \neq 0$, then we have $\Phi^{+}(x m) \geq \Phi^{+}(m)$ and $\Phi^{-}(x m) \leq \Phi^{-}(m)$. Now

$$
\begin{aligned}
\Phi^{+} & \left(m_{1}-m_{2}\right) \cdot \gamma=\mathrm{m}_{\gamma}\left(\Phi^{+}\right)\left(m_{1}-m_{2}\right) \\
& \geq \min \left\{\mathrm{m}_{\gamma}\left(\Phi^{+}\right)\left(m_{1}\right), \mathrm{m}_{\gamma}\left(\Phi^{+}\right)\left(m_{2}\right)\right\} \\
& =\min \left\{\Phi^{+}\left(m_{1}\right) \cdot \gamma, \Phi^{+}\left(m_{2}\right) \cdot \gamma\right\} \\
& =\min \left\{\Phi^{+}\left(m_{1}\right), \Phi^{+}\left(m_{2}\right)\right\} \cdot \gamma,
\end{aligned}
$$

which means that $\Phi^{+}\left(m_{1}-m_{2}\right) \geq \min \left\{\Phi^{+}\left(m_{1}\right), \Phi^{+}\left(m_{2}\right)\right\}$.

Similarly, $\Phi^{-}\left(m_{1}-m_{2}\right) \leq \max \left\{\Phi^{-}\left(m_{1}\right), \Phi^{-}\left(m_{2}\right)\right\}$. Hence $\Phi=\left(M ; \Phi^{+}, \Phi^{-}\right)$is a bipolar fuzzy BCK-submodule on $M$.

The following theorem provides a strong relationship between $(\alpha, \beta)$-translation and $(\gamma, \delta)$-multiplication of any bipolar fuzzy BCK-submodule.

Theorem 28. Let $\Phi=\left(M ; \Phi^{+}, \Phi^{-}\right)$be a bipolar fuzzy set on $M,(\alpha, \beta) \in[0, T] \times[\perp, 0]$, and $\gamma, \delta \in(0,1]$. Then $T_{(\alpha, \beta)}(\Phi)$ is a bipolar fuzzy s-extension of $\mathrm{m}_{(\gamma, \delta)}(\Phi)$.

Proof. For every $m \in M$, we have

$$
\begin{aligned}
T_{\alpha}\left(\Phi^{+}\right)(m) & =\Phi^{+}(m)+\alpha \geq \Phi^{+}(m) \geq \Phi^{+}(m) \cdot \gamma \\
& =\mathrm{m}_{\gamma}\left(\Phi^{+}\right)(m), \\
T_{\beta}\left(\Phi^{-}\right)(m) & =\Phi^{-}(m)+\beta \leq \Phi^{-}(m) \leq \Phi^{-}(m) \cdot \delta \\
& =\mathrm{m}_{\delta}\left(\Phi^{-}\right)(m),
\end{aligned}
$$

and so $T_{(\alpha, \beta)}(\Phi)$ is a bipolar fuzzy extension of $\mathrm{m}_{(\gamma, \delta)}(\Phi)$.

Now let $\mathrm{m}_{(\gamma, \delta)}(\Phi)$ be a bipolar fuzzy BCK-submodule on $M$, where $\gamma, \delta \in(0,1]$. Then $\Phi$ is a bipolar fuzzy BCKsubmodule on $M$ by Theorem 27. It follows from Theorem 15 that the $(\alpha, \beta)$-translation $T_{(\alpha, \beta)}(\Phi)$ of $\Phi$ is a bipolar fuzzy BCK-submodule on $M$ for all $(\alpha, \beta) \in[0, T] \times[\perp, 0]$. Therefore every $(\alpha, \beta)$-translation $T_{(\alpha, \beta)}(\Phi)$ of $\Phi$ is a bipolar fuzzy $s$-extension of the $(\gamma, \delta)$-multiplication $\mathrm{m}_{(\gamma, \delta)}(\Phi)$ of $\Phi$.

The following example shows that Theorem 28 is not valid for $\gamma=0$ (or $\delta=0)$.

Example 29. Consider a BCK-algebra $X=\{0, a, b, c, d\}$ and $X$-module $M=\{0, a, b, c\}$ that are defined in Example 19. on $M$

Equation (37) defines a bipolar fuzzy set $\Phi=\left(M ; \Phi^{+}, \Phi^{-}\right)$

\begin{tabular}{|c|c|c|c|c|}
\hline$M$ & 0 & $a$ & $b$ & $c$ \\
\hline$\Phi^{+}$ & 0.8 & 0.7 & 0.6 & 0.3 \\
\hline$\Phi^{-}$ & -0.9 & -0.6 & -0.8 & -0.6 \\
\hline
\end{tabular}

If we take $\gamma=0$, then the $(\gamma, \delta)$-multiplication is a bipolar fuzzy BCK-submodule on $M(\forall \delta \in(0,1])$ since

$$
\begin{aligned}
\mathrm{m}_{0} & \left(\Phi^{+}\right)\left(m_{1}-m_{2}\right)=0 \\
= & \min \left\{\mathrm{m}_{0}\left(\Phi^{+}\right)\left(m_{1}\right), \mathrm{m}_{0}\left(\Phi^{+}\right)\left(m_{2}\right)\right\},
\end{aligned}
$$




$$
\begin{aligned}
& \mathrm{m}_{0}\left(\Phi^{+}\right)(x m)=0=\mathrm{m}_{0}\left(\Phi^{+}\right)(m) \\
& \mathrm{m}_{\delta}\left(\Phi^{-}\right)\left(m_{1}-m_{2}\right)=\Phi^{-}\left(m_{1}-m_{2}\right) \cdot \delta \\
& \quad \leq \max \left\{\Phi^{-}\left(m_{1}\right), \Phi^{-}\left(m_{2}\right)\right\} \cdot \delta, \\
& \mathrm{m}_{\delta}\left(\Phi^{-}\right)(x m)=\Phi^{-}(x m) \cdot \delta \leq \Phi^{-}(m) \cdot \delta \\
& \quad=\mathrm{m}_{\delta}\left(\Phi^{-}\right)(m),
\end{aligned}
$$

for all $m, m_{1}, m_{2} \in M$ and $x \in X$. But if we take $m_{1}=a$ and $m_{2}=b$, then $T_{\alpha}\left(\Phi^{+}\right)(a-b)=\Phi^{+}(a-b)+\alpha=\Phi^{+}(c)+\alpha=$ $0.3+\alpha<0.6+\alpha=\min \left\{\Phi^{+}(a), \Phi^{+}(b)\right\}+\alpha$ for all $\alpha \in[0,0.2]$ and so $T_{(\alpha, \beta)}(\Phi) \notin \mathscr{B} \mathscr{F} \mathcal{S}(M)$. Hence $T_{(\alpha, \beta)}(\Phi)$ is not a bipolar fuzzy $s$-extension of $\mathrm{m}_{(0, \delta)}(\Phi)$ for all $\in[0,0.2], \beta \in[-0.1,0]$.

Corollary 30. Let $f: M \rightarrow N$ be a homomorphism of BCKmodules and $\Psi=\left(N ; \Psi^{+}, \Psi^{-}\right)$a bipolar fuzzy set on $N$. If $\Psi$ is a bipolar fuzzy BCK-submodule, then so is $f^{-1}\left(T_{(\alpha, \beta)}(\Psi)\right)$ for any $(\alpha, \beta)$-translation $T_{(\alpha, \beta)}(\Psi)$ of $\Psi$ with $(\alpha, \beta) \in[0, T] \times[\perp, 0]$.

Proof. Proof is directly by Theorems 12 and 15. lary.

Joining Theorems 13 and 16 we have the following corol-

Corollary 31. Let $f: M \rightarrow N$ be an epimorphism of BCK-modules and $\Psi=\left(N ; \Psi^{+}, \Psi^{-}\right)$a bipolar fuzzy set on $N$. If the inverse image $f^{-1}\left(T_{(\alpha, \beta)}(\Psi)\right)=\left(M ; f^{-1}\left(T_{\alpha}\left(\Psi^{+}\right)\right)\right.$, $\left.f^{-1}\left(T_{\beta}\left(\Psi^{-}\right)\right)\right)$of the $(\alpha, \beta)$-translation is a bipolar fuzzy BCKsubmodule for some $(\alpha, \beta) \in[0, \top] \times[\perp, 0]$, then so is $\Psi$.

Corollary 32. Let $f: M \rightarrow N$ be an epimorphism of $B C K$-modules and $\Phi=\left(M ; \Phi^{+}, \Phi^{-}\right) \in \mathscr{B} \mathscr{F} \mathcal{S}(M)$. Then for any $(\alpha, \beta)$-translation $T_{(\alpha, \beta)}(\Phi)$ of $\Phi$, the homomorphic image $f\left(T_{(\alpha, \beta)}(\Phi)\right) \in \mathscr{B} \mathscr{F} \mathcal{S}(N)$.

Using Theorems 10,12, 13, and 27, we deduce the following results.

Corollary 33. Let $f: M \rightarrow N$ be a homomorphism of BCKmodules and $\Psi=\left(N ; \Psi^{+}, \Psi^{-}\right)$a bipolar fuzzy BCK-submodule on $N$, and then the inverse image $f^{-1}\left(\mathrm{~m}_{(\gamma, \delta)}(\Psi)\right) \in \mathscr{B} \mathscr{F} \mathcal{S}(M)$ for any $(\gamma, \delta)$-multiplication of $\Psi$ with $\gamma, \delta \in[0,1]$.

Corollary 34. Let $f: M \rightarrow N$ be an epimorphism of BCKmodules. If $f^{-1}\left(\mathrm{~m}_{(\gamma, \delta)}(\Psi)\right) \in \mathscr{B} \mathscr{F} \mathcal{S}(M)$ for a bipolar fuzzy set $\Psi=\left(N ; \Psi^{+}, \Psi^{-}\right)$and some $\gamma, \delta \in(0,1]$, then $\Psi \in \mathscr{B} \mathscr{F} \mathcal{S}(N)$.

Corollary 35. Let $f: M \rightarrow N$ be an epimorphism of BCKmodules and $\Phi=\left(M ; \Phi^{+}, \Phi^{-}\right) \in \mathscr{B} \mathscr{F} \mathcal{S}(M)$, and then $f\left(\mathrm{~m}_{(\gamma, \delta)}(\Phi)\right) \in \mathscr{B} \mathscr{F} \mathcal{S}(N)$ for any $\gamma, \delta \in(0,1]$.

\section{Conclusion}

Translations and multiplications are types of operators attractive to researchers who are interested in fuzzy set theory. Generally, main objective of studying translations and multiplications is to inspect whether a certain form of fuzzy set remains invariant under such operators. In this paper, we showed that a bipolar fuzzy BCK-submodule keeps its property under translations and multiplications. Further, we presented a relation between translations and multiplications of bipolar fuzzy BCK-submodules. More properties and results were provided.

\section{Competing Interests}

The authors declare that there is no conflict of interests regarding the publication of this paper.

\section{References}

[1] L. A. Zadeh, "Fuzzy sets," Information and Control, vol. 8, pp. 338-353, 1965

[2] K. M. Lee, "Bipolar-valued fuzzy sets and their operations," in Proceedings of the International Conference on Intelligent Technologies, pp. 307-312, Bangkok, Thailand, 2000.

[3] K. J. Lee, "Bipolar fuzzy subalgebras and bipolar fuzzy ideals of BCK/BCI-algebras," Bulletin of the Malaysian Mathematical Sciences Society, vol. 32, no. 3, pp. 361-373, 2009.

[4] M. Zhou and S. Li, "Applications of bipolar fuzzy theory to hemirings," International Journal of Innovative Computing, Information and Control, vol. 10, no. 2, pp. 767-781, 2014.

[5] M. Zhou and S. Li, "Application of bipolar fuzzy sets in semirings," Journal of Mathematical Research with Applications, vol. 34, no. 1, pp. 61-72, 2014.

[6] H. A. S. Abujabal, M. Aslam, and A. B. Thaheem, "On actions of BCK-algebras on groups," Panamerican Mathematical Journal, vol. 4, no. 3, pp. 43-48, 1994.

[7] M. Bakhshi, "Fuzzy set theory applied to BCK-modules," Advances in Fuzzy Sets and Systems, vol. 2, no. 8, pp. 61-87, 2011.

[8] M. M. Altaiary, S. A. Bashammakh, and N. O. Alshehri, " $\epsilon$ , $\epsilon, v q)$-Fuzzy BCK-Submodules," Discrete Dynamics in Nature and Society, vol. 2016, Article ID 8746275, 7 pages, 2016.

[9] Y. B. Jun, H. S. Kim, and K. J. Lee, "Bipolar fuzzy translations in BCK/BCI-algebras," Journal of Chungcheong Mathematical Society, vol. 3, no. 22, pp. 399-408, 2009.

[10] K. J. Lee, Y. B. Jun, and M. I. Doh, "Fuzzy translations and fuzzy multiplications of BCK/BCI-algebras," Communications of the Korean Mathematical Society, vol. 24, no. 3, pp. 353-360, 2009.

[11] Y. B. Jun, "Translations of fuzzy ideals in BCK/BCI-algebras," Hacettepe Journal of Mathematics and Statistics, vol. 40, no. 3, pp. 349-358, 2011.

[12] A. Kashif and M. Aslam, "Homology theory of BCK-modules," Southeast Asian Bulletin of Mathematics, vol. 38, no. 1, pp. 61-72, 2014. 


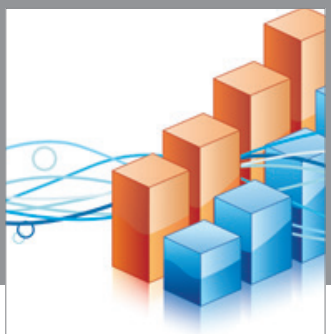

Advances in

Operations Research

vatem alat4

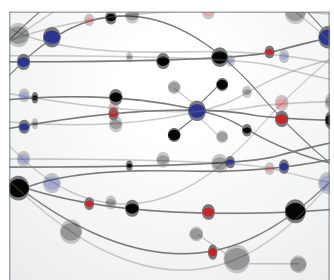

\section{The Scientific} World Journal
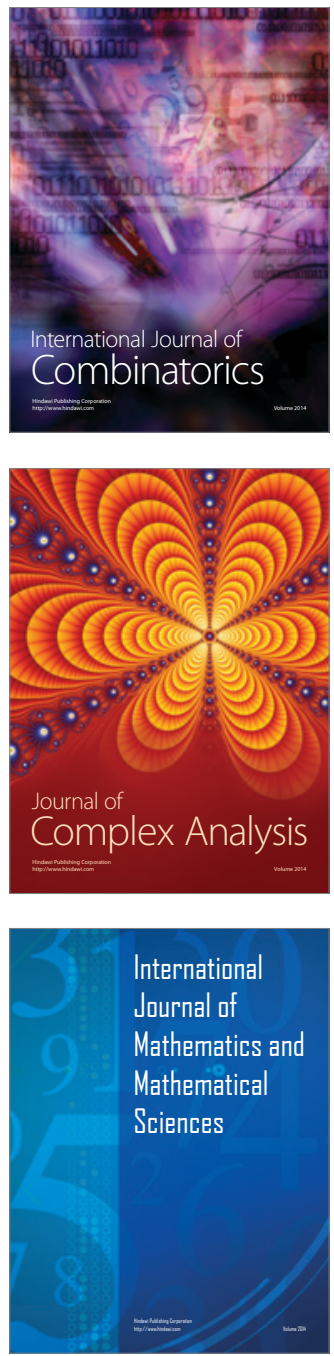
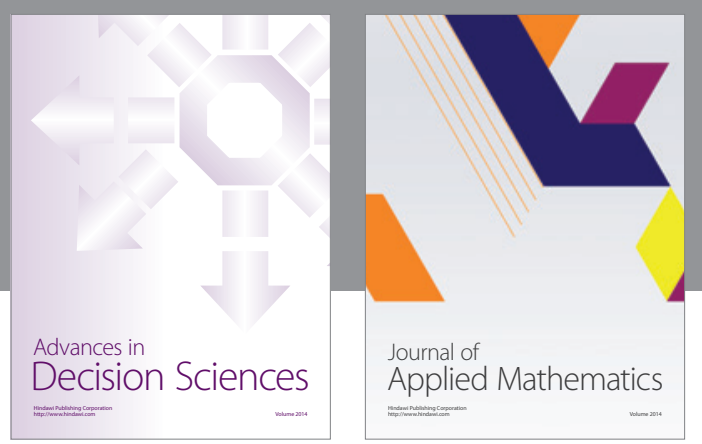

Algebra

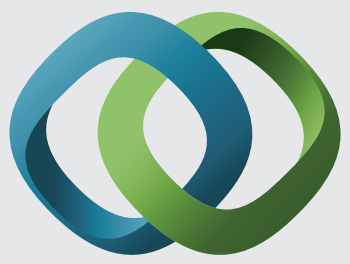

\section{Hindawi}

Submit your manuscripts at

https://www.hindawi.com
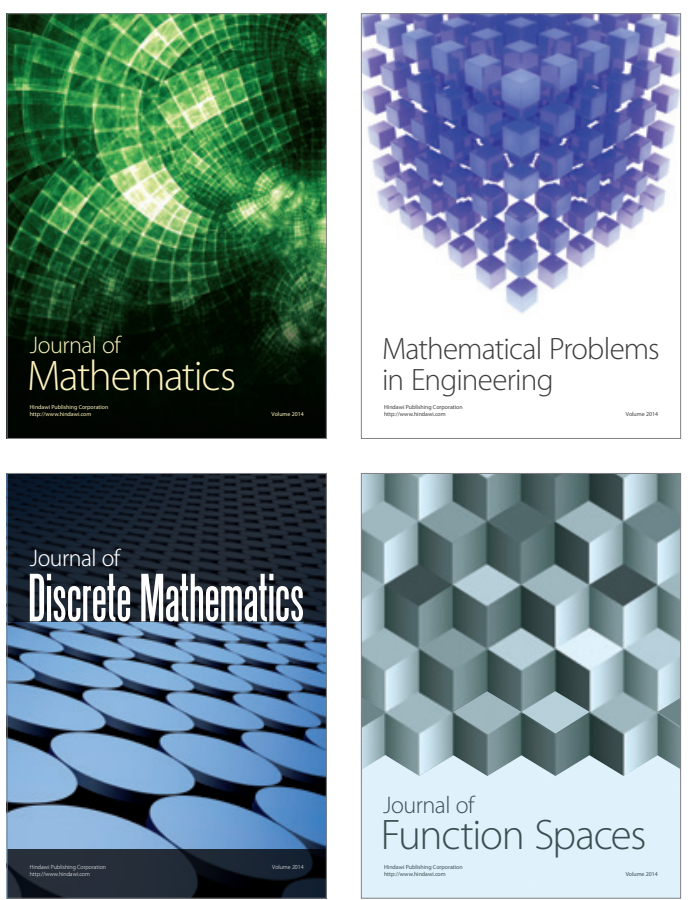

Mathematical Problems in Engineering
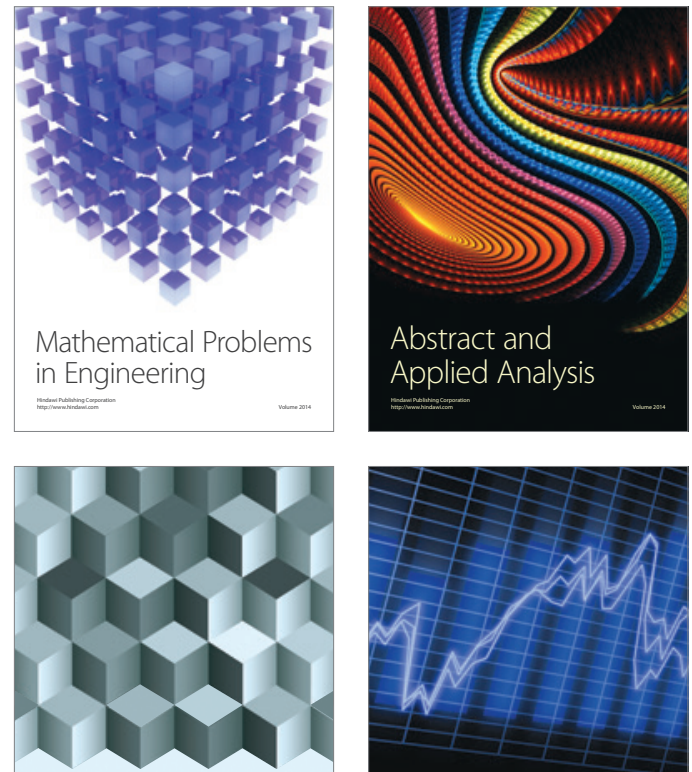

Journal of

Function Spaces

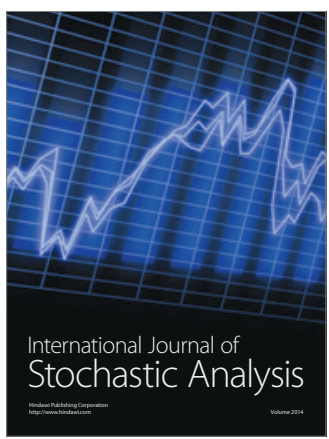

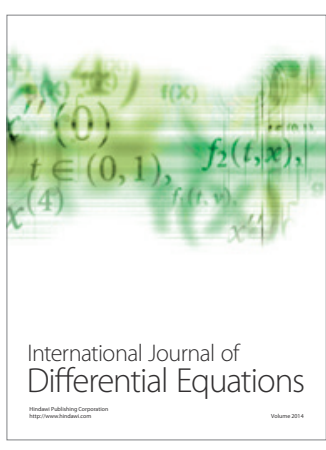
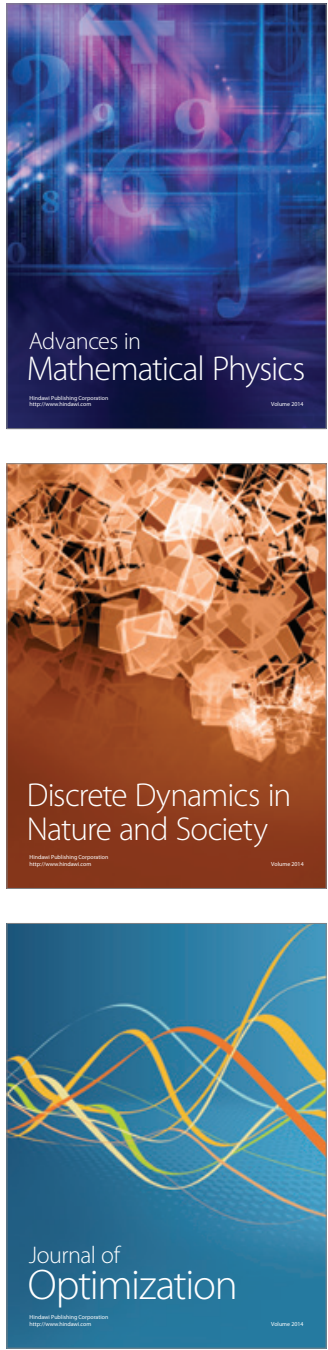